\section{MINI- REVIEW}

\title{
Phenotypic heterogeneity: differential stress resistance among individual cells of the yeast Saccharomyces cerevisiae
}

\author{
Edward R. Sumner and Simon V. Avery
}

\author{
Author for correspondence: Simon V. Avery. Tel: +44 115 9513315. Fax: +44 1159513251. \\ e-mail: Simon.Avery@nottingham.ac.uk
}

School of Life and Environmental Sciences, University of Nottingham, University Park, Nottingham NG7 2RD, UK

Keywords: cell-to-cell variability, flow cytometry, cell cycle, ageing, non-genetic variation

\section{Overview}

Phenotypic heterogeneity describes non-genetic variation that exists between individual cells within isogenic populations. Such heterogeneity is readily evident in the differential sensitivity to stress of genetically identical cells and can be fundamental to the fitness and persistence of an organism. Consequently, phenotypic heterogeneity is currently receiving increased attention from the scientific community. Here, we present the first review of this important subject, with an account that focuses on the factors that contribute to cell-to-cell diversity in the model eukaryotic micro-organism Saccharomyces cerevisiae. Some of the key parameters that drive non-genetic heterogeneity include cell cycle progression, cell ageing, mitochondrial activity, epigenetic regulation and potentially also stochastic variation. The relative significance of these and other parameters in generating heterogeneity is discussed. We refer in particular to differential stress sensitivity in S. cerevisiae, although other relevant phenomena such as phenotypic switching in Candida spp. are also addressed.

\section{Background}

Classical genetics (e.g. gene mutation or overexpression) has helped to identify genes whose absence or presence in a cell influences phenotype. However, these approaches do not explain the phenotypic heterogeneity that is evident among individual cells within isogenic cultures. Cells within isogenic populations exhibit broad phenotypic variation. For experimental purposes, such variation is readily apparent in phenotypes that are selectable. For example, where only $\sim 50 \%$ of cells in a pure culture are competent for growth after an environmental insult, what differed between those cells at the moment of exposure that determined which died and which survived? Almost any study that has used measurements of \% viability (or other similar parameter) to help ascertain phenotype will have documented such intermediate killing - i.e. other than $0 \%$ or $100 \%$ - and this is indicative of cell-to-cell variation. Yet, in only a few cases has such variation been investigated in detail. This is surprising since nongenetic heterogeneity can be fundamental to the fitness of an organism, particularly where there is little genetic heterogeneity. There are many situations in which individual species commonly exist isogenically (e.g. in industrial fermentations, food, laboratory cultures, human infection, etc.), and more so when considering organisms' complements of genes that influence phenotype. Hence the determinants of phenotypic heterogeneity can be of far greater relevance to real problems such as stress in industrial or laboratory settings for S. cerevisiae - than the factors uncovered by the study of engineered variants. So phenotypic diversity may be critical for the persistence of an organism during stress, as exemplified in bacteria by the occurrence of antibiotic persisters (Davis et al., 1990) and in spoilage yeast by acid-resistant 'super cells' (Steels et al., 2000).

That there have been few direct studies of phenotypic heterogeneity to date in part reflects the inability to purify phenotypically divergent subpopulations for study by subculture; since the determinants of phenotypic heterogeneity are not usually inherited, such subpopulations again generally show variable phenotype after subculture (Davis et al., 1990; Steels et al., 2000). Thus characterization of phenotypic variants requires growth-independent means of differentiation at the single-cell level. Techniques such as flow cytometry provide the necessary tools for these analyses (Howlett \& Avery, 1999; Attfield et al., 2001).

S. cerevisiae is an excellent candidate for studies of phenotypic heterogeneity since it commonly exists in isogenic populations, particularly in settings relevant to 
human use (see above), and its experimental tractability and widespread adoption as a eukaryotic model are well documented. Thus several recent studies of phenotypic heterogeneity have focused on S. cerevisiae, and this organism provides the main focus for this mini-review.

The potential mechanisms underlying phenotypic heterogeneity have been speculated upon in several papers. The purpose of this mini-review is to address each of the possible cell variables that may contribute to nongenetic heterogeneity and to discuss the roles of these variables in determining differential stress resistance.

\section{Genetic versus non-genetic variation in 'isogenic' cultures}

Pure cultures in liquid medium are usually derived from single clonal colonies. Spontaneous mutations that arise during culturing can only account for a small fraction of cell-to-cell variation. For example, after 5 days outgrowth of a single diploid yeast cell with a $2 \mathrm{~h}$ doubling time, approximately 1 in 175 cells might harbour a spontaneous mutation that affects fitness (Zeyl \& DeVisser, 2001), with a markedly smaller proportion being affected for any single phenotype. Although the frequency of spontaneous genetic variation may be elevated through loss of certain gene functions (e.g. those causing mutator phenotypes), such contributions still cannot account for the extent of variation that is evident in young clonal cultures, e.g. where $50 \%$ of the population might exhibit one phenotype and 50\% another.

The simplest way to discriminate experimentally whether a phenotype is genetically determined is to test for inheritance of the phenotype in progeny. Of all the possible factors (see sections below) that might determine non-genetic resistance of a fraction of cells to a short-term stress, none, with the possible exception of epigenetic regulation, are sustainable through the multiple rounds of cell division and physiological changes associated with growth. However, if maintained with stressor, one way in which such cells could appear to sustain resistance during growth would be if initial survival allowed them time to mount an adaptive response, i.e. induction of genes whose products conferred resistance. Such inducible resistance would still be lost on removal of the stressor however. Therefore, only genetically determined phenotypes should be inherited indefinitely in the absence of selection. In this way, rare reversion to oxytetracycline resistance in sod1 $S$. cerevisiae was shown to be genetic (Avery et al., 2000), whereas sorbic-acid resistance of some cells in Zygosaccharomyces bailii cultures was non-genetic (Steels et al., 2000).

Thus phenotypic heterogeneity is not related to differential gene possession. However, it could arise from differential basal gene expression. Heterogeneity in basal gene expression could be linked to most of the cell variables discussed in the following sections. It is stressed that genes revealed to influence a phenotype through comparison of non-isogenic organisms (e.g. mutant versus wild-type) are only potential contributors to heterogeneity in a phenotype. True determinants of phenotypic heterogeneity are decisive, in a non-engineered system.

\section{Cell division cycle}

A principal cell variable in exponential phase cultures is cell cycle stage. Thus individual cells are distributed between $G_{1}, S, G_{2}$ and $M$ cell cycle stages, in approximate proportion to the durations of these stages. Cell cycle progression is associated with marked fluctuations in the transcriptional activity of many yeast genes (Spellman et al., 1998) and also in gross cell characteristics such as cell volume (see below). Traditionally, prior arrest with cell-cycle-blocking agents or with cell division cycle $(c d c)$ mutations has been used to assess whether a phenotype is cell-cycle-dependent. Thus $G_{1}$-arrested $S$. cerevisiae cultures were more resistant to the superoxide-generating agent menadione than non-arrested cultures, but less resistant to hydrogen peroxide (Flattery-O’Brien \& Dawes, 1998). $\mathrm{G}_{2}$-arrested cells showed the reverse phenotypes. The intermediate degree of resistance evident in heterogeneous nonarrested cultures would reflect a mean of the differing cell states. Leroy et al. (2001) have shown that $\mathrm{H}_{2} \mathrm{O}_{2}$ sensitivity in S-phase $S$. cerevisiae may be a result of ineffective DNA base excision repair during this stage. Of course, agents that directly target cell cycle functions will by definition also elicit heterogeneous toxicity in exponential populations, as has been substantiated with arrested or synchronized cultures (Nitiss \& Wang, 1996; Schmitt et al., 1996).

One problem with using cell cycle arrest to establish the cell cycle dependency of a phenotype is that synchronization methods commonly exert secondary perturbing effects on cell physiology. Flow cytometry can be used to circumvent this problem, and provides an excellent tool for examining cell-to-cell heterogeneity generally. In our laboratory, flow cytometry has been used to demonstrate that heterogeneous sensitivity to $\mathrm{Cu}$ in exponential-phase $S$. cerevisiae is at least partly cell-cycledependent (Howlett \& Avery, 1999). Cells in the $G_{2} / M$ phases of the cell cycle were predominant in $\mathrm{Cu}$-sensitive subpopulations, whereas $G_{1} / S$-phase cells were predominant in Cu-resistant subpopulations (Fig. 1). Furthermore, with the use of an oxidant-sensitive fluorescent probe in conjunction with flow cytometry, it was established that initial (pre-Cu exposure) cellular oxidant status was predictive of $\mathrm{Cu}$ sensitivity (Howlett $\&$ Avery, 1999).

The mechanisms underlying cell-cycle-dependent changes in cellular oxidant status and in other phenotypes are unknown in many cases. Changes in gene transcriptional activity could underpin many such phenotypes. Tests of this possibility should be facilitated by the available listing of all cell-cycle-regulated yeast genes (Spellman et al., 1998), together with the development of fluorescent reporters for monitoring dynamically ex- 

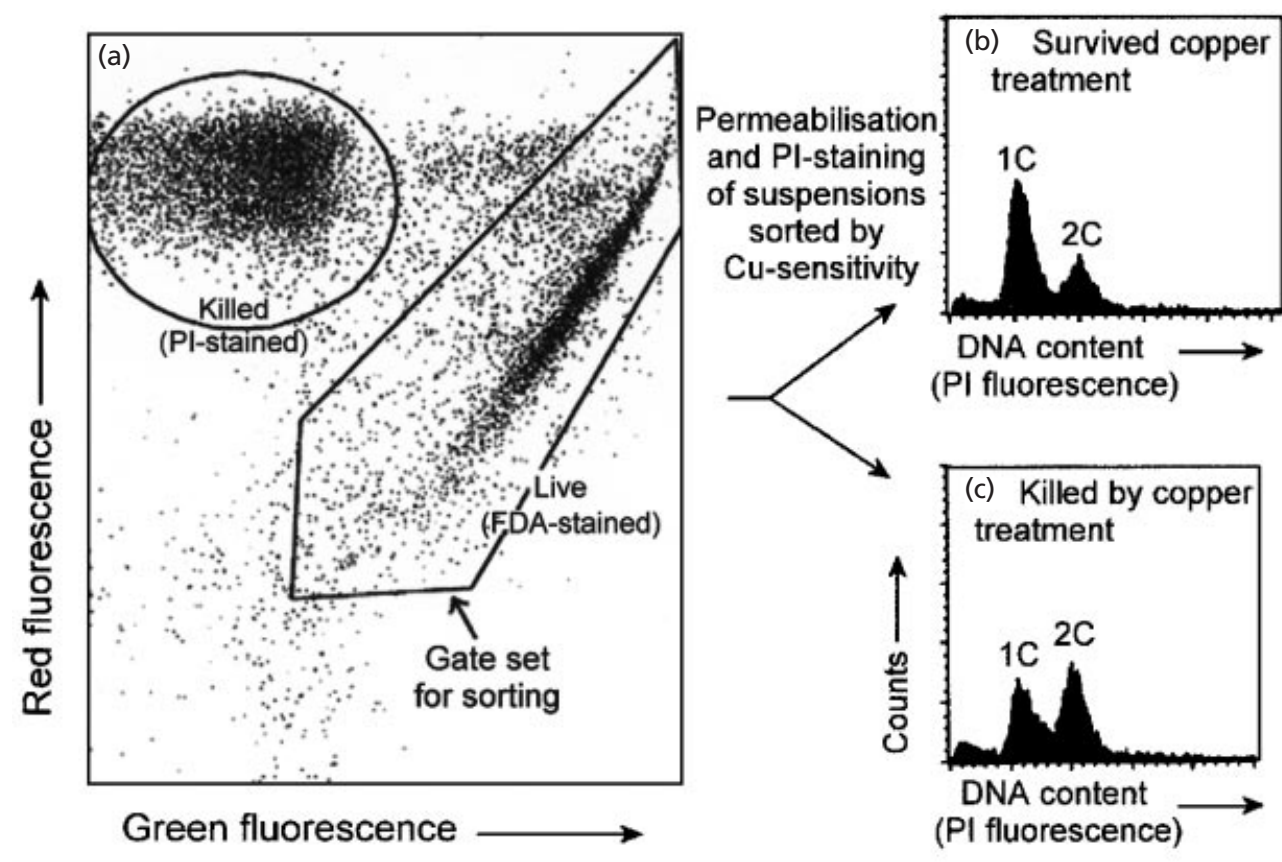

Fig. 1. Cell cycle dependency of $\mathrm{Cu}$ sensitivity in S. cerevisiae, determined with flow cytometry/cell sorting. Viable and non-viable cells after 5 min $\mathrm{Cu}\left(\mathrm{NO}_{3}\right)_{2}$ exposure (a), and DNA contents of sorted viable cells (b) and non-viable cells (c). PI, Propidium iodide; FDA, fluorescein diacetate. 1C DNA content (pre DNA replication) pertains to $G_{1} / S$ phase cells, and $2 \mathrm{C}$ DNA pertains to $G_{2} / M$ cells. Adapted from Howlett \& Avery (1999) with permission from Elsevier Science.

pressed yeast genes (Mateus \& Avery, 2000), of strategies for simultaneous determination of stress resistance and gene expression (Attfield et al., 2001), and of microarray hybridization techniques. More general parameters (e.g. cell volume) could also effect cell-cycledependent phenotypic heterogeneity and these are included among the factors addressed below.

\section{Rhythms}

Ultradian rhythms are cellular biological cycles with a period shorter than $1 \mathrm{~d}$, which become readily synchronized (and so discernible) during continuous culture of $S$. cerevisiae. Usually, such oscillations in S. cerevisiae are also synchronized with the cell division cycle, although cell-cycle-independent oscillation has been reported (Keulers et al., 1996). In standard cultures, cells will be asynchronous with regard to ultradian rhythmicity. Using continuous cultures synchronized with an ultradian rhythm of $\sim 3-4 \mathrm{~h}$, Wang et al. (2000) showed that cellular resistances to $\mathrm{H}_{2} \mathrm{O}_{2}$, menadione and heat all oscillated, and did so in phase with each other. Resistance to cadmium and 1-chloro-2,4-dinitrobenzene (an uncoupler of mitochondrial metabolism) also oscillated, with a peak advanced to that of the other stressors. The GTS1 gene product helps to establish these oscillations (Wang et al., 2001), so GTS1 must also help mediate regulation of the factors (e.g. stress resistance genes) that affected stress resistance directly. Clearly, outputs of biological clocks can contribute to cellular diversity in exponential cultures, and perhaps independently of the cell cycle.

\section{Growth rate of individual cells and cell size}

The sizes and growth rates of individual cells in $S$. cerevisiae cultures are highly heterogeneous. This heterogeneity is linked primarily to the asymmetric cell division of budding yeast and to ageing: newly budded daughter cells are usually smaller and usually display longer generation times than the parent cells from which they were derived. In addition, the sizes of daughter cells are variable. Smaller daughter cells reside for a longer period in the $G_{1}$, pre-Start, phase of the cell cycle than larger daughters, which results in a longer doubling time of the smaller daughters (Brewer et al., 1984). The reverse relationship may hold for parent cells, which exhibit increased mean volume but also a prolonged generation time as they age, particularly in cells nearing the end of their replicative life span (see Cell age section). These effects may account for some complex cell-to-cell heterogeneity in yeast cultures (see Fig. 2) (for more detailed accounts, also see Wheals \& Lord, 1992; Kennedy et al., 1994).

Enhanced resistance to lytic enzymes and to heat shock has been demonstrated in slowly growing yeast cultures (Elliott \& Futcher, 1993). The authors of this work argued that such resistance was a property of slow growth per se and was at least partly independent of other factors such as cell cycle phase (though unbudded cells were slightly more resistant than budded cells in some experiments). It follows that the variability in individual cell growth rate (and size) that occurs within yeast cultures can account for at least some of the heterogeneity in stress sensitivity that is evident. This 
Cell cycle stage / ultradian phase / cell size

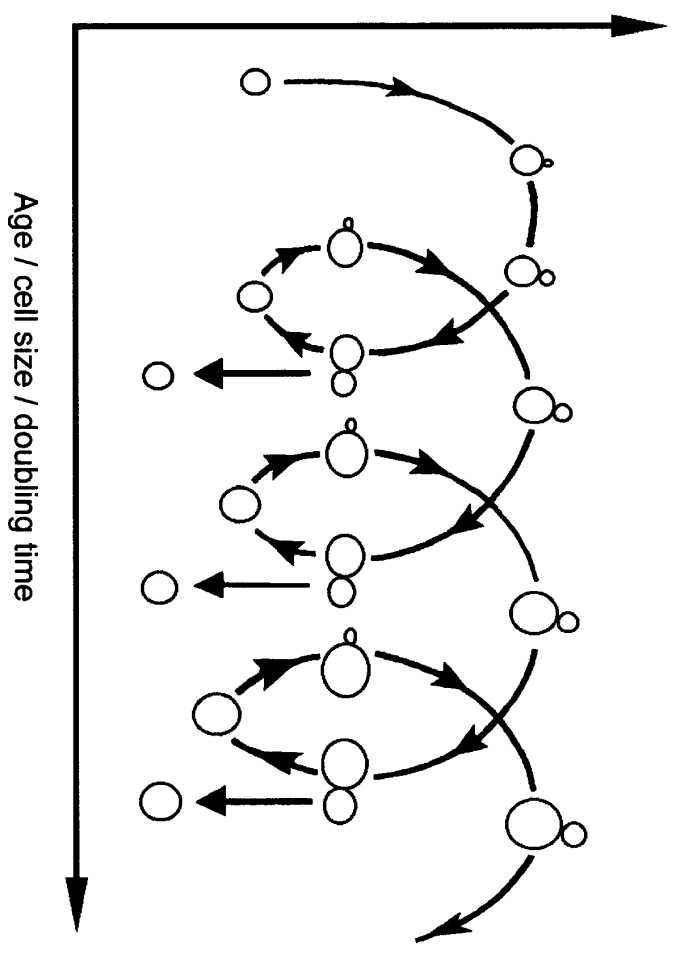

Fig. 2. Adaptation of the $S$. cerevisiae cell spiral of Jazwinski (1996) to show general cell parameters that change as cells progress through the cell cycle and as they age.

has not been tested directly, which must partly relate to the difficulty of dissecting experimentally the influences of cell age and cell cycle away from those of cell size and growth rate. The potential molecular mechanisms underlying size- and growth-rate-dependent effects on stress sensitivity are unknown and would be difficult to elucidate since parameters such as cell-surface to volume ratio exert such broad effects on cell physiology.

\section{Cell age}

$S$. cerevisiae divides a finite number of times before senescence, so yeast cell age is often determined as a generation number (i.e. the number of times a cell has given rise to a daughter) rather than in terms of absolute chronological age. The proportion of old cells in a typical population declines exponentially as a function of generation number and therefore young cells heavily predominate. For example, virgin cells that have never divided comprise roughly $50 \%$ of the total, whereas tenth-generation cells represent less than $0.05 \%$ of the population. Thus any phenotype specific to cells, say, ten generations or older, should affect only a small proportion of the population, whereas phenotypes specific to younger cells would exert a broader (but not necessarily more significant) influence on the whole population.

A range of physiological changes is associated with ageing in S. cerevisiae. Gross parameters such as cell size and generation time are influenced by cell age (discussed above), as well as more specific phenotypes many of which may arise from differential gene transcription that occurs during ageing of $S$. cerevisiae (Jazwinski, 1996). Stress sensitivity is affected by cell age. Kale \& Jazwinski (1996) prepared purified cells of different ages and compared their resistances to the DNA-alkylating agent ethyl methanosulphate (EMS) and to UV irradiation. Susceptibility to EMS increased steadily with age, whereas resistance to UV peaked in 'middle-aged' cells (approx. eight generations old) and declined markedly in older cells. This biphasic pattern of UV resistance was closely correlated with the level of RAS2 mRNA; Ras $2 \mathrm{p}$ is involved in the repair of UV-induced DNA damage (but not EMS-induced DNA damage). The authors concluded that age-dependent changes in stress sensitivity were related directly to the ageing process rather than incidental changes in cell physiology (e.g. via cell volume changes). It was suggested that age-dependent variation in stress sensitivity might have arisen to ensure persistence of clonal populations against naturally occurring stresses such as UV irradiation. Such a scenario would be in keeping with the proposed general role of phenotypic heterogeneity in enabling the persistence of isogenic populations (see Background section).

\section{Mitochondrial function}

The mitochondrial activity of individual yeast cells in a population is variable. Petite mutants can arise spontaneously and are characterized by a partial or total loss of mitochondrial DNA and consequent inability to respire (they grow by fermentation). A fraction of cells in exponential yeast cultures are usually phenotypically petite. There may also be significant differences in the mitochondrial activity of grande (mitochondria-containing) cells within cultures. Mitochondrial numbers in grande cells generally vary between one to ten in cycling cultures, with highly dynamic changes owing to rapid mitochondrial division and fusion events (Jensen et al., 2000). Furthermore, mitochondrial DNA replication occurs throughout the cell cycle such that mitochondrial genome copy number also is variable (Sena et al., 1975). In addition, heterogeneity may reside in the activity of individual mitochondria. The membrane potential of mitochondria in mammalian cells is quite variable (Salvioli et al., 2000) and flow cytometric analyses do not discount that this may also be the case for yeast mitochondria (Ludovico et al., 2001).

The influence of mitochondrial activity on yeast phenotype can be readily assessed by comparison of petite and grande cultures. Altered stress responses in petite cells have been widely reported, although such phenotypes can be strain-specific. Altered susceptibility may be related to certain of the parameters discussed elsewhere in this review. For example, owing to their fermentative metabolism, petite mutants generally display slow growth rates. Moreover, mitochondrial activity is directly related to the generation of highly damaging reactive oxygen species (ROS, which are mostly free radicals) that are formed as by-products of respiration. 
Many types of stress promote ROS action or formation in cells. For example, freeze-thaw stress is considered to increase cytosolic ROS activity in yeast via an oxidative burst of superoxide radicals formed from oxygen and electrons leaked from the mitochondrial electron transport chain (Park et al., 1998). Metals such as $\mathrm{Cu}, \mathrm{Cd}$ and $\mathrm{Cr}$ also may promote the production of ROS in a mitochondria-dependent manner (Avery, 2001). Petite mutants actually exhibit enhanced susceptibility to certain types of free-radical-generating stressors, and this has been attributed to lack of some energygenerating capability that may be required for active free radical detoxification (Grant et al., 1997).

In addition to the above effects, a pathway termed the 'retrograde response' signals mitochondrial dysfunction to the nucleus in S. cerevisiae and results in modulation of the nuclear gene expression profile. A recent microarray analysis of a petite strain entirely lacking mitochondrial DNA revealed increased expression of heatshock proteins and members of the ABC family of drug transporters. Accordingly, the mutant was resistant to severe heat shock and to several drugs (Traven et al., 2001).

Thus the presence of petite cells in normal yeast cultures and the heterogeneous mitochondrial activity of grande cells together have the potential to generate considerable heterogeneity in cell phenotypes that are influenced by mitochondrial function. Such heterogeneity should be absent in petite cultures.

\section{Epigenetic regulation and prions}

Any heritable factor that affects the phenotype of an organism but that does not act through alteration of the genotype is said to be regulated epigenetically. In eukaryotes, one form of epigenetic regulation involves the formation of protein-DNA structures termed heterochromatin at certain chromosomal domains. In S. cerevisiae, heterochromatin forms at several chromosomal regions, including the telomeres, and is metastably inherited in the progeny. Genes adjacent to regions of heterochromatin are transcriptionally silenced. For example, when ADE2 is inserted close to telomeres silencing results in the formation of redcoloured colonies, but loss of ADE2 silencing is evident from the occasional development of white colonysectors. Thus spontaneous loss of transcriptional silencing can produce distinct subpopulations (Thompson et al., 1993). The relevance of such epigenetic regulation to differential stress sensitivity in S. cerevisiae is not yet known. However, any genes that influence stress sensitivity and that are located in chromosomal domains subject to silencing, for example, should have the potential to give rise to stress-sensitive and -insensitive cell subpopulations. Progeny would generally inherit the phenotypes of such subpopulations, unlike phenotypes associated with the other cell-to-cell variables that are reviewed here.

Although the focus of this review is on S. cerevisiae, in the context of epigenetic regulation of phenotypic variation in yeasts it is worth addressing the important subject of phenotypic switching that occurs in certain Candida spp., including pathogenic Candida albicans (for a review, see Soll, 1997). There is evidence that phenotypic switching may serve as a virulence factor in C. albicans. Such phenotypic switching is readily observed as changes in colony appearance that may typically occur at frequencies in the order of 1 in $10^{4}$. Moreover, switching may be accompanied by a broad range of phenotypic changes at the single cell level also (e.g. antigen expression, tissue affinity). It is thought that heritable but reversible changes in heterochromatic state and/or possible chromosomal rearrangements underpin these phenotypic switches. Thus deletion of the SIR 2 gene that is involved in maintaining chromatin silencing yielded a switching frequency in C. albicans as high as 1 in 10 (Perez-Martin et al., 1999).

A protein-based form of epigenetic inheritance involves the so-called yeast prions. Prion-like proteins exist in two heritable conformational states - 'normal' and 'prion'. Much recent work has focused on a prion element that results from aggregation of the Sup35 translation termination factor in $S$. cerevisiae. Conversion from the normal form, $\left[p s i^{-}\right]$, to prion, $\left[\mathrm{PSI}^{+}\right]$, is self-perpetuating and may result in reduced fidelity of protein synthesis and enhanced variation at the proteome level (Pal, 2001). Recent studies have revealed that $\left[\mathrm{PSI}^{+}\right]$strains exhibit altered susceptibility to a wide array of stresses compared to co-isogenic $\left[p s i^{-}\right]$strains (Eaglestone et al., 1999; True \& Lindquist, 2000). For example, in roughly half of over 150 different environmental conditions tested, significant differences in growth rate were noted between co-isogenic $\left[\mathrm{PSI}^{+}\right]$and $\left[p s i^{-}\right]$strains, and $\left[\mathrm{PSI}^{+}\right]$strains showed enhanced growth relative to $\left[\right.$ psi $\left.^{-}\right]$strains in over $25 \%$ of these tests (True \& Lindquist, 2000). It has been suggested that $\left[\mathrm{PSI}^{+}\right]$-induced variability may be selectively maintained in yeast as a means of enhancing the survival of clonal populations in the event of adverse environmental conditions (True \& Lindquist, 2000). However, the presence of prions can explain only limited phenotypic variation. Prions do not appear to be widespread and in $S$. cerevisiae may be restricted to laboratory strains; prion-forming ability has not been identified in natural or industrial strains (Chernoff et al., 2000). Furthermore, as with spontaneous mutation (see above), the frequencies of changes in phenotype of $\left[\mathrm{PSI}^{+}\right]$cells and of spontaneous conversion between normal and prion states (True \& Lindquist, 2000) are too low to account for much of the phenotypic heterogeneity evident within yeast cultures.

\section{Stochastic variation}

Regulatory gene networks involving a broad range of transcription factors and other signalling molecules can control cellular processes. Many signalling molecules are present in very small copy numbers per cell. Through signal amplification, random fluctuations in the concentrations of these factors have the potential to lead ultimately to the adoption of distinct fates in individual 
cells (McAdams \& Arkin, 1999). Some pathogenic bacteria can exploit this stochastic variation to generate phenotypic diversity in clonal populations; this enables some cells to escape recognition by the host's immune system. McAdams \& Arkin (1997) proposed that many previously unexplained cases of phenotypic diversity in isogenic cell populations might be explained by stochastic variation at the level of gene regulation. Indeed, stochastic variation seems likely to impinge on several of the processes discussed elsewhere in this review (e.g. replicative life span, cell size in newly budded cells). Nevertheless, the direct contribution of stochastic effects to the differential stress sensitivity of $S$. cerevisiae cells could be relatively small. Redundancy has evolved between different genes and signalling pathways of cells such that if the outcome of one pathway would lead to an unfavourable cellular response, other pathways can act to compensate for this failing (Kerszberg, 1996). Also, in situations where stochastic variation is quite transient, cellular outcomes arising from signalling pathways may be more appropriate than if no stochastic variation was present at all: this phenomenon has been termed 'stochastic focusing' and acts to reduce the uncertainty in regulated processes (Paulsson et al., 2000).

\section{Concluding remarks}

There have still been only a few detailed studies of the factors responsible for phenotypic heterogeneity, yet such heterogeneity determines the fates of individual cells in isogenic populations and probably the ultimate fates of populations themselves. Cell cycle stage, cell age, cell growth rate, cell rhythms, prion elements and mitochondrial activity are all non-genetic variables that influence yeast cell sensitivity to one or more stresses. Other epigenetic factors and stochastic variation also are likely to play some role. The relative importance of these various parameters in generating heterogeneity is likely to be phenotype-specific. Nevertheless, based on the evidence to date, cell cycle progression and cell ageing together with attendant changes in cell size, etc., and mitochondrial activity seem to be among the most dominant factors that drive heterogeneous stress sensitivity. The molecular mechanisms that underpin such heterogeneity (e.g. that determine cell-cycle- or growthrate-dependent phenotypes) in most cases remain to be elucidated.

\section{Acknowledgements}

Work in the Avery laboratory is supported by grants from the National Institutes of Health (R01 GM57945), the Royal Society (22233) and the University of Nottingham Research Committee.

\section{References}

Attfield, P. V., Choi, H. Y., Veal, D. A. \& Bell, P. J. L. (2001). Heterogeneity of stress gene expression and stress resistance among individual cells of Saccharomyces cerevisiae. Mol Microbiol 40, 1000-1008.

Avery, S. V. (2001). Metal toxicity in yeasts and the role of oxidative stress. Adv Appl Microbiol 49, 111-142.
Avery, S. V., Malkapuram, S., Mateus, C. \& Babb, K. S. (2000). Copper/zinc superoxide dismutase is required for oxytetracycline resistance of Saccharomyces cerevisiae. J Bacteriol 182, 76-80.

Brewer, B. J., Chlebowicz-Sledziewska, E. \& Fangman, W. L. (1984). Cell cycle phases in the unequal mother/daughter cell cycles of Saccharomyces cerevisiae. Mol Cell Biol 4, 2529-2531.

Chernoff, Y. O., Galkin, A. P., Lewitin, E., Chernova, T. A., Newnam, G. P. \& Belenkiy, S. M. (2000). Evolutionary conservation of prion-forming abilities of the yeast Sup35 protein. Mol Microbiol 35, 865-876.

Davis, B. D., Dulbecco, R., Eisen, H. N. \& Ginsberg, H. S. (1990). Microbiology, 4th edn. Philadelphia: J. B. Lippincott.

Eaglestone, S. S., Cox, B. S. \& Tuite, M. F. (1999). Translation termination efficiency can be regulated in Saccharomyces cerevisiae by environmental stress through a prion-mediated mechanism. EMBO J 18, 1974-1981.

Elliott, B. \& Futcher, B. (1993). Stress resistance of yeast cells is largely independent of cell cycle phase. Yeast 9, 33-42.

Flattery-O'Brien, J. A. \& Dawes, I. W. (1998). Hydrogen peroxide causes $R A D$ 9-dependent cell cycle arrest in $\mathrm{G}_{2}$ in Saccharomyces cerevisiae whereas menadione causes $G_{1}$ arrest independent of RAD9 function. J Biol Chem 273, 8561-8571.

Grant, C. M., Maclver, F. H. \& Dawes, I. W. (1997). Mitochondrial function is required for resistance to oxidative stress in the yeast Saccharomyces cerevisiae. FEBS Lett 410, 219-222.

Howlett, N. G. \& Avery, S. V. (1999). Flow cytometric investigation of heterogeneous copper-sensitivity in asynchronously grown Saccharomyces cerevisiae. FEMS Microbiol Lett 176, 379-386.

Jazwinski, S. M. (1996). Longevity, genes and aging. Science 273, 54-59.

Jensen, R. E., Hobbs, A. E. A., Cerveny, K. L. \& Sesaki, H. (2000). Yeast mitochondrial dynamics: fusion, division, segregation, and shape. Microsc Res Tech 51, 573-583.

Kale, S. P. \& Jazwinski, S. M. (1996). Differential response to UV stress and DNA damage during the yeast replicative life span. Dev Genet 18, 154-160.

Kennedy, B. K., Austriaco, N. R. \& Guarente, L. (1994). Daughter cells of Saccharomyces cerevisiae from old mothers display a reduced life span. J Cell Biol 127, 1985-1993.

Kerszberg, M. (1996). Accurate reading of morphogen concentrations by nuclear receptors: a formal model of complex transduction pathways. J Theor Biol 183, 95-104.

Keulers, M., Satroutdinov, A. D., Suzuki, T. \& Kuriyama, H. (1996). Synchronization affector of autonomous short-periodsustained oscillation of Saccharomyces cerevisiae. Yeast 12, 673-682.

Leroy, C., Mann, C. \& Marsolier, M. C. (2001). Silent repair accounts for cell cycle specificity in the signalling of oxidative DNA lesions. EMBO J 20, 2896-2906.

Ludovico, P., Sansonetty, F. \& Côrte-Real, M. (2001). Assessment of mitochondrial membrane potential in yeast cell populations by flow cytometry. Microbiology 147, 3335-3343.

McAdams, H. H. \& Arkin, A. (1997). Stochastic mechanisms in gene expression. Proc Natl Acad Sci U S A 94, 814-819.

McAdams, H. H. \& Arkin, A. (1999). It's a noisy business! Genetic regulation at the nanomolar scale. Trends Genet 15, 65-69.

Mateus, C. \& Avery, S. V. (2000). Destabilized green fluorescent protein for monitoring dynamic changes in yeast gene expression with flow cytometry. Yeast 16, 1313-1323.

Nitiss, J. L. \& Wang, J. C. (1996). Mechanisms of cell killing by 
drugs that trap covalent complexes between DNA topoisomerases and DNA. Mol Pharmacol 50, 1095-1102.

Pal, C. (2001). Yeast prions and evolvability. Trends Genet 17, 167-169.

Park, J. I., Grant, C. M., Davies, M. J. \& Dawes, I. W. (1998). The cytoplasmic $\mathrm{Cu}, \mathrm{Zn}$ superoxide dismutase of Saccharomyces cerevisiae is required for resistance to freeze-thaw stress generation of free radicals during freezing and thawing. $J$ Biol Chem 273, 22921-22928.

Paulsson, J., Berg, O. G. \& Ehrenberg, M. (2000). Stochastic focusing: fluctuation-enhanced sensitivity of intracellular regulation. Proc Natl Acad Sci US A 97, 7148-7153.

Perez-Martin, J., Uria, J. A. \& Johnson, A. D. (1999). Phenotypic switching in Candida albicans is controlled by a SIR2 gene. EMBO J 18, 2580-2592.

Salvioli, S., Dobrucki, J., Moretti, L., Troiano, L., Fernandez, M. G., Pinti, M., Pedrazzi, J., Franceschi, C. \& Cossarizza, A. (2000). Mitochondrial heterogeneity during staurosporine-induced apoptosis in HL60 cells : analysis at the single cell and single organelle level. Cytometry 40, 189-197.

Schmitt, M. J., Klavehn, P., Wang, J., Schonig, I. \& Tipper, D. J. (1996). Cell cycle studies on the mode of action of yeast K28 killer toxin. Microbiology 142, 2655-2662.

Sena, E. P., Welch, J. W., Halvorson, H. O. \& Fogel, S. (1975). Nuclear and mitochondrial deoxyribonucleic acid replication during mitosis in Saccharomyces cerevisiae. J Bacteriol 123, 497-504.

Soll, D. R. (1997). Gene regulation during high-frequency switching in Candida albicans. Microbiology 143, 279-288.
Spellman, P. T., Sherlock, G., Zhang, M. Q., lyer, V. R., Anders, K., Eisen, M. B., Brown, P. O., Botstein, D. \& Futcher, B. (1998). Comprehensive identification of cell cycle-regulated genes of the yeast Saccharomyces cerevisiae by microarray hybridization. Mol Biol Cell 9, 3273-3297.

Steels, H., James, S. A., Roberts, I. N. \& Stratford, M. (2000). Sorbic acid resistance: the inoculum effect. Yeast 16, 1173-1183.

Thompson, J. S., Hecht, A. \& Grunstein, M. (1993). Histones and the regulation of heterochromatin in yeast. Cold Spring Harbor Symp Quant Biol 58, 247-256.

Traven, A., Wong, J. M. S., Xu, D., Sopta, M. \& Ingles, J. C. (2001). Altered nuclear gene expression profiles in a yeast mitochondrial DNA mutant. J Biol Chem 276, 4020-4027.

True, H. L. \& Lindquist, S. L. (2000). A yeast prion provides a mechanism for genetic variation and phenotypic diversity. Nature 407, 477-483.

Wang, J. Q., Liu, W. D., Uno, T., Tonozuka, H., Mitsui, K. \& Tsurugi, K. (2000). Cellular stress responses oscillate in synchronization with the ultradian oscillation of energy metabolism in the yeast Saccharomyces cerevisiae. FEMS Microbiol Lett 189, 9-13.

Wang, J. Q., Liu, W. D., Mitsui, K. \& Tsurugi, K. (2001). Evidence for the involvement of the GTS1 gene product in the regulation of biological rhythms in the continuous culture of the yeast Saccharomyces cerevisiae. FEBS Lett 489, 81-86.

Wheals, A. E. \& Lord, P. G. (1992). Clonal heterogeneity in specific growth rate of Saccharomyces cerevisiae cells. Cell Prolif 25, 217-223.

Zeyl, C. \& DeVisser, J. A. G. M. (2001). Estimates of the rate and distribution of fitness effects of spontaneous mutation in Saccharomyces cerevisiae. Genetics 157, 53-61. 